\title{
A GROWTH PROPERTY OF THE NEVANLINNA CHARACTERISTIC
}

\section{ALLEN WEITSMAN ${ }^{1}$}

ABSTRACr. We prove here a relation between the rate of growth of the Nevanlinna characteristic of a meromorphic function $f(z)$, the size of a deficient value $\tau$, and the sets where $f(z)$ is close to $\tau$.

1. Introduction. In the study of the distribution of values of meromorphic functions $f(z)$, one finds that the size of the Nevanlinna deficiency of a value, the measure of the subsets of certain circumferences on which the function is close to the value, and the rate of growth of the Nevanlinna characteristic (as determined for example by its order or lower order) are all interdependent. The purpose of this note is to give an elementary insight into this connection.

Let $f(z)$ be a nonconstant meromorphic function in $|z|<\infty$. In addition to the familiar symbols of Nevanlinna's theory

$$
\begin{gathered}
n(r, f), \quad N(r, f), \quad m(r, f), \\
T(r, f), \quad \delta(\tau, f),
\end{gathered}
$$

and the order $\lambda$ and lower order $\mu$ defined by

$$
\begin{aligned}
& \lambda=\limsup _{r \rightarrow \infty} \frac{\log T(r, f)}{\log r}, \\
& \mu=\liminf _{r \rightarrow \infty} \frac{\log T(r, f)}{\log r},
\end{aligned}
$$

we shall use the notation

$$
E(r)=\left\{\theta: 0 \leqq \theta<2 \pi,\left|f\left(r e^{i \theta}\right)\right|>1\right\},
$$

and denote by $C E(r)$ the complement of $E(r)$ with respect to $[0,2 \pi)$. We define a sequence $\rho_{m} \rightarrow \infty$ to be a $\gamma$-sequence if

$$
\limsup _{m \rightarrow \infty} \frac{\rho_{m} T^{\prime}\left(\rho_{m}, f\right)}{T\left(\rho_{m}, f\right)} \leqq \gamma
$$

Received by the editors August 10, 1969.

AMS 1969 subject classifications. Primary 3060, 3061.

Key words and phrases. Nevanlinna characteristic, deficient value, meromorphic function.

1 Research supported by NSF grant GP-9454. 
where $T^{\prime}$ denotes the derivative of $T(r, f)$ with respect to $r$. Since $T(r, f)$ is differentiable for all $r>r_{0}$ (see for example $[1$, p. 626]) and

$$
r T^{\prime}(r, f) / T(r, f)=d \log T(r, f) / d \log r
$$

it is clear from the definitions (1.1) that there exists a $\gamma$-sequence for every $\mu \leqq \gamma \leqq \lambda$.

We shall prove the following

THEOREM. Let $f(z)$ be a nonconstant meromorphic function, $\left\{\rho_{m}\right\}$ a $\gamma$-sequence, and $E(r)$ as in (1.2). Then, if $\delta(\infty, f)>0$ we have

$$
\liminf _{m \rightarrow \infty} \operatorname{meas} E\left(\rho_{m}\right) \geqq 4 \operatorname{ctn}^{-1}\left(\frac{\pi \gamma}{\delta(\infty, f)}\right) .
$$

In proving Theorem 1 we shall prove an inequality slightly better than (1.4). In fact, taking

$$
s_{\infty}=\liminf _{m \rightarrow \infty} \operatorname{meas} E\left(\rho_{m}\right)
$$

we shall prove

$$
\pi \gamma \geqq \delta(\infty, f) \operatorname{ctn}\left(\frac{s_{\infty}}{4}\right)+\delta(0, f) \operatorname{ctn}\left(\frac{2 \pi-s_{\infty}}{4}\right)
$$

from which (1.4) is apparent. (The inequality (1.6) remains valid in the extreme cases $s_{\infty}=0$ or $s_{\infty}=2 \pi$ so long as we adopt the convention $0 \cdot \infty=0$.)

It is perhaps worth pointing out that more sophisticated methods of this nature have been developed in recent years by A. Edrei [3] where the sequence $\left\{\rho_{m}\right\}$ is replaced by a different well-chosen sequence $\left\{r_{m}\right\}$, the "Polya peaks of order $\mu$ " of the function (cf. [2], [3], [6]). It seems, however, that the methods developed thus far regarding the quantities $E\left(r_{m}\right)$ and those developed here for $E\left(\rho_{m}\right)$ yield results which are not best possible. In the case of the Polya peaks, Edrei has conjectured $[4$, p. 57] that

$$
\liminf _{m \rightarrow \infty} \operatorname{meas} E\left(r_{m}\right) \geqq \min \left(\frac{4}{\mu} \sin ^{-1}\left(\frac{\delta(\infty, f)}{2}\right)^{1 / 2}, 2 \pi\right) .
$$

2. Proof of (1.6). Set

$$
P(R, r, \theta, \phi)=\frac{1}{2 \pi} \frac{R^{2}-r^{2}}{R^{2}-2 R r \cos (\theta-\phi)+r^{2}} \quad(r<R)
$$


and recall the familiar properties of the Poisson kernel

$$
\begin{gathered}
\int_{0}^{2 \pi} P(R, r, \theta, \phi) d \theta=1, \\
P(R, r, \theta, \phi)>0, \\
P(R, r, \theta, \phi)=P(R, r, \phi, \theta) .
\end{gathered}
$$

It is without loss of generality that we suppose

$$
\begin{aligned}
& f(z) \neq 0, \infty \\
& \quad \text { for } 0 \leqq \rho_{m}-|z| \leqq \epsilon_{m}\left(\epsilon_{m}>0\right), m=1,2, \cdots .
\end{aligned}
$$

In fact, since it is an elementary exercise to show that meas $E(r)$ and $r T^{\prime}(r, f) / T(r, f)$ are continuous functions of $r>r_{0}$ (cf. [1, p. 626]), we may always choose a $\gamma$-sequence "close to" the sequence $\left\{\rho_{m}\right\}$ for which (2.4) is satisfied, and having the same value for $s_{\infty}$ in (1.5).

In what follows, we set

$$
g(R, r, \psi, w)=\log \left|\frac{R^{2}-\bar{w} r e^{i \psi}}{R\left(r e^{i \psi}-w\right)}\right| .
$$

Now, for $0<\rho_{m}-\rho \leqq \epsilon_{m}$, by (1.2), (2.1), (2.3), and the PoissonJensen formula we may write

$$
\begin{aligned}
& \frac{\rho_{m}\left(m\left(\rho_{m}, f\right)-m(\rho, f)\right)}{\rho_{m}-\rho} \\
& =\frac{\rho_{m}}{\rho_{m}-\rho}\left(\frac{1}{2 \pi} \int_{E\left(\rho_{m}\right)} \log \left|f\left(\rho_{m} e^{i \theta}\right)\right| \int_{0}^{2 \pi} P\left(\rho_{m}, \rho, \phi, \theta\right) d \phi d \theta\right. \\
& \\
& \quad-\frac{1}{2 \pi} \int_{E(\rho)} \int_{0}^{2 \pi} \log \left|f\left(\rho_{m} e^{i \theta}\right)\right| P\left(\rho_{m}, \rho, \theta, \phi\right) d \theta d \phi \\
& +\frac{1}{2 \pi} \sum_{f\left(a_{\nu}\right)=0 ;\left|a_{\nu}\right|<\rho_{m}} \int_{E(\rho)} g\left(\rho_{m}, \rho, \theta, a_{\nu}\right) d \theta \\
& \left.\quad-\frac{1}{2 \pi} \sum_{f\left(b_{\nu}\right)=\infty ;\left|b_{\nu}\right|<\rho_{m}} \int_{E(\rho)} g\left(\rho_{m}, \rho, \theta, b_{\nu}\right) d \theta\right) .
\end{aligned}
$$

Interchanging the order of integration in the second integral and writing $\Sigma_{0}$ and $\Sigma_{\infty}$ for the two sums in the preceding line we have 
68

ALLEN WEITSMAN

[September

$$
\begin{aligned}
& \frac{\rho_{m}\left(m\left(\rho_{m}, f\right)-m(\rho, f)\right)}{\rho_{m}-\rho} \\
& =\frac{\rho_{m}}{\rho_{m}-\rho}\left(\frac{1}{2 \pi} \int_{E\left(\rho_{m}\right)} \log \left|f\left(\rho_{m} e^{i \theta}\right)\right| \int_{0}^{2 \pi} P\left(\rho_{m}, \rho, \phi, \theta\right) d \phi d \theta\right. \\
& \quad-\frac{1}{2 \pi} \int_{0}^{2 \pi} \log \mid f\left(\rho_{m} e^{i \theta)} \mid \int_{E(\rho)} P\left(\rho_{m}, \rho, \phi, \theta\right) d \phi d \theta\right. \\
& =\frac{\rho_{m}}{2 \pi} \int_{E\left(\rho_{m}\right)} \log \mid f\left(\rho_{m} e^{i \theta) \mid} \int_{C E(\rho)} \frac{P\left(\rho_{m}, \rho, \phi, \theta\right) d \phi}{\rho_{m}-\rho} d \theta\right. \\
& \quad+\frac{\rho_{m}}{2 \pi} \int_{C E\left(\rho_{m}\right)} \log \frac{1}{\left|f\left(\rho_{m} e^{i \theta}\right)\right|} \int_{E(\rho)} \frac{P\left(\rho_{m}, \rho, \phi, \theta\right) d \phi}{\rho_{m}-\rho} d \theta \\
& \quad+\frac{\rho_{m}}{2 \pi\left(\rho_{m}-\rho\right)} \Sigma_{0} \\
& \quad-\frac{\rho_{m}}{2 \pi\left(\rho_{m}-\rho\right)} \Sigma_{\infty} .
\end{aligned}
$$

Now, an elementary computation (cf. [8, p. 17]), (2.1), and (2.2) yield

$$
\begin{aligned}
\frac{\rho_{m}}{2 \pi} \Sigma \int_{E(\rho)} \frac{g\left(\rho_{m}, \rho, \theta, a_{v}\right)}{\rho_{m}}-\rho & \\
& \rightarrow \Sigma \int_{E(\rho)} P\left(\rho_{m},\left|a_{\nu}\right|, \theta, \arg a_{\nu}\right) d \theta>0
\end{aligned}
$$

and

$$
\begin{aligned}
n\left(\rho_{m}, f\right)-\frac{\rho_{m}}{2 \pi} & \Sigma \int_{E(\rho)} \frac{g\left(\rho_{m}, \rho, \theta, b_{\nu}\right)}{\rho_{m}-\rho} d \theta \\
& \rightarrow \Sigma \int_{C E(\rho)} P\left(\rho_{m},\left|b_{\nu}\right|, \theta, \arg b_{\nu}\right) d \theta>0
\end{aligned}
$$

as $\boldsymbol{\rho} \rightarrow \rho_{m}$.

Also, it is evident that 


$$
\begin{aligned}
& \frac{\rho_{m}}{2 \pi\left(\rho_{m}-\rho\right)} \int_{E(\rho)} \frac{\left(\rho_{m}-\rho\right)\left(\rho_{m}+\rho\right) d \phi}{\rho_{m}^{2}-2 \rho_{m} \rho \cos (\theta-\phi)+\rho^{2}} \\
& \quad \geqq \frac{\rho_{m}}{\pi} \int_{(1 / 2) \text { meas } C E(\rho) \rho_{m}^{2}-2 \rho_{m} \rho \cos \beta+\rho^{2}}^{\pi} \frac{\left(\rho_{m}+\rho\right) d \beta}{\pi} \\
& \quad \rightarrow \frac{1}{\pi} \int_{(1 / 2) \text { meas } C E\left(\rho_{m}\right)}^{\pi} \frac{d \beta}{4} \cos \beta \\
&=\frac{1}{\pi} \operatorname{ctn} \frac{\text { meas } C E\left(\rho_{m}\right)}{4} \quad\left(\rho \rightarrow \rho_{m}\right) .
\end{aligned}
$$

Add $\rho_{m} N^{\prime}\left(\rho_{m}, f\right)\left(=n\left(\rho_{m}, f\right)\right)$ to both sides of $(2.6)$ and let $\rho \rightarrow \rho_{m}$. After dividing by $T\left(\rho_{m}, f\right)$, and applying (1.3), (2.7), (2.8), (2.9), and an inequality similar to (2.9) for $C E(\rho)$, we obtain (1.6).

3. Some consequences of (1.6). Suppose $f(z)$ is a meromorphic function having at least two deficient values

$$
\delta\left(\tau_{1}, f\right)=\delta_{1}>0, \quad \delta\left(\tau_{2}, f\right)=\delta_{2}>0 .
$$

By taking a Möbius transformation of $f(z)$ which sends $\tau_{1}$ into 0 and $\tau_{2}$ into $\infty$ we deduce from (1.6) that the transformed function cannot have a 0 -sequence and thus must have positive lower order. Since $f(z)$ has the same lower order it must again be positive. This fact has been obtained previously by another method [5, p. 297]. The fact that a meromorphic function of order zero cannot have two deficient values was established earlier by Valiron [9].

Similarly, by the same considerations as above, the first fundamental theorem of Nevanlinna's theory, and the mean value theorem, we find that if $f(z)$ has two deficient values (3.1) then there exists $\eta=\eta\left(\delta_{1}, \delta_{2}\right)>0$ such that

$$
\liminf _{r \rightarrow \infty} \frac{T(\sigma r, f)}{T(r, f)} \geqq \sigma^{\eta}
$$

for every $\sigma \geqq 1$.

\section{BIBLIOGRAPHY}

1. H. Cartan, Sur la derivée par rapport à log $r$ de la fonction de croissance $T(r ; f)$, C. R. Acad. Sci. Paris 189 (1929), 625-627.

2. A. Edrei, The deficiencies of meromorphic functions of finite lower order, Duke Math. J. 31 (1964), 1-21. MR 28 \#1306. 
3. - Sums of deficiencies of meromorphic functions, J. Analyse Math. 14 (1965), 79-107. MR 31 \#4909.

4. - Sums of deficiencies of meromorphic functions. II, J. Analyse Math. 19 (1967), 53-74. MR $35 \# 6831$.

5. A. Edrei and W. Fuchs, On the growth of meromorphic functions with several deficient values, Trans. Amer, Math. Soc. 93 (1959), 292-328. MR 22 \#770.

6. - The deficiencies of meromorphic functions of order less than one, Duke Math. J. 27 (1960), 233-249. MR 23 \#A1039.

7. W. Hayman, Meromorphic functions, Math. Monographs, Clarendon Press, Oxford, 1964. MR 29 \#1337.

8. Z. Nehari, Conformal mapping, McGraw-Hill, New York, 1952. MR 13, 640.

9. G. Valiron, Sur les valeurs déficientes des fonctions méromorphes d'ordre nul, C. R. Acad. Sci. Paris 230 (1950), 40-42. MR 11, 344.

Purdue University, Lafayette, Indiana 47907 